Forschende

Komplementärmedizin und

Klassische Naturheilkunde

\section{Editorial}

Forsch Komplementärmed Klass Naturheilkd 2001;8:193-195

\title{
Das Wirksamkeitsparadox in der Komplementärmedizin
}

Überblicksarbeiten über komplementärmedizinische Interventionen kommen nicht selten zu dem verwirrenden Schluss, dass sich die Intervention als wirksam erwiesen habe im Vergleich zu Nichtbehandlung und Wartegruppen oder gar als gleich wirksam wie etablierte Therapien, aber nicht wirksamer als Placebo. Dies liegt zum Teil daran, dass manche Typen von Studien wenig durchgeführt werden. Ein anderer Grund für diesen Befund könnte aber auch das von mir so benannte Wirksamkeitsparadox sein. Hinter diesem Begriff verbirgt sich folgender Sachverhalt, den ich in einem kurzen Gedankenexperiment schildern will (Abb. 1).

Stellen wir uns zwei Behandlungen für eine Krankheit vor, sagen wir chronischen Schmerz, die in zwei placebokontrollierten Studien getestet wurden, bei vergleichbaren Patientenpopulationen, gemessen mit denselben Instrumenten. Behandlung y, so nehmen wir an, habe sich als wirksam erwiesen, Behandlung $\mathrm{x}$ dagegen nicht, und zwar weil im Vergleich zwischen Behandlung y und Placebo y die Behandlungsgruppe der Placebogruppe signifikant überlegen war, Behandlung $\mathrm{x}$ aber nicht gegenüber Placebo $\mathrm{x}$. Behandlung $\mathrm{x}$, so wollen wir einmal annehmen, zeigte nämlich nur eine Überlegenheit von $10 \%$ gegenüber der Placebokontrolle, Behandlung y jedoch eine Überlegenheit von $20 \%$, ein Unterschied, der bei den vorhandenen Patientenzahlen signifikant war. Die Schlussfolgerung der Autoren in einer Publikation über die Studienergebnisse des Vergleichs von Behandlung $\mathrm{x}$ gegenüber Placebo $\mathrm{x}$ wären: «x ist unwirksam». Die Schlussfolgerung beim Vergleich von y gegenüber Placebo y hingegen wären: «y ist wirksam». Jeder, der die Grafik auch nur flüchtig betrachtet, sieht, dass mit dieser Argumentation etwas im Argen liegt. Worauf beruhen die Schlussfolgerungen, und was bleibt unberücksichtigt? Die Schlussfolgerungen beruhen einzig und allein auf dem relativen Unterschied zwischen Verumbehandlung und Placebobehandlung, der die Grösse des spezifischen Effekts der Verumbehandlung abbilden soll. Dieser spezifische Effekt, so nimmt man an, ist derjenige Anteil am Therapieerfolg, der aufgrund der «eigentlichen», spezifischen Wirksamkeit der Intervention zustande kommt, wohingegen die unspezifischen
Therapieeffekte sich in der Placebogruppe niederschlagen. $\mathrm{Zu}$ diesen gehören verschiedene Effekte, unter anderem Messartefakte aufgrund der Ungenauigkeit der Messinstrumente, statistische Regression zur Mitte, natürlicher Krankheitsverlauf und unspezifische Effekte, die zustande kommen, weil Patienten eine Besserung erwarten, da ihnen im Rahmen einer Behandlung mehr Aufmerksamkeit zuteil wird; im Rahmen dieser Behandlung sind sie Teil einer speziellen Studie mit den entsprechenden Ehrfurcht einflössenden Ritualen, die alle Besserung verheissen. Artefakte, Regressionseffekte und unspezifische Therapieeffekte gemeinsam machen das aus, was man gemeinhin den Placeboeffekt im Rahmen einer klinischen Studie nennt. Messartefakte und Regressionseffekte sind, das dürfen wir annehmen, in beiden hypothetischen Studien gleich gross. Denn die Messartefakte hängen einzig und allein von der Art der verwendeten Masse ab, und diese setzen wir als gleich voraus. Regressionseffekte sind bedingt durch die Art der behandelten Krankheit und die Charakteristik der Patientenpopulation, die wir ebenfalls als gleich voraussetzen. Die unspezifischen Therapieeffekte jedoch, so wollen wir annehmen, sind variabel und hängen ab vom Setting der Therapie und einer Fülle von Faktoren, die teilweise beim Patienten zu suchen sind - Erwartungen, Hoffnungen, Veränderung des Lebensstils, Diät oder Verhaltensweisen -, teilweise beim Therapeuten - Fähigkeit, eine tragfähige Beziehung aufzubauen, Vermittlung von Zuversicht usw. In unserem Gedankenexperiment machen diese unspezifischen Effekte in Behandlung x $30 \%$ des Gesamteffekts aus, in Behandlung y jedoch nur $5 \%$. Diese hypothetische Situation führt nun dazu, dass Behandlung $\mathrm{x}$ zwar absolut gesehen viel erfolgreicher ist, weil durch sie $70 \%$ aller Patienten Besserung erfahren, wogegen in Behandlung y nur 55\% gebessert werden. Aber das Urteil der Forschung erklärt $\mathrm{x} z u$ einer unwirksamen, $\mathrm{y}$ jedoch $\mathrm{zu}$ einer wirksamen Therapie.

Wie kommt dieses Paradox zustande? Offensichtlich dadurch, dass man stillschweigend annimmt, dass die Artefakte und unspezifischen Effekte allgemein gleich gross und deswegen vernachlässigbar sind, und überdies, dass nur die spezifischen

\begin{tabular}{ll}
\hline KARGER & $\oplus$ 2001 S. Karger GmbH, Freiburg \\
$\begin{array}{l}\text { Fax }+497614520714 \\
\begin{array}{l}\text { E-mail Information@Karger.de } \\
\text { www.karger.com }\end{array}\end{array}$ & $\begin{array}{l}\text { Accessible online at: } \\
\text { www.karger.com/journals/fkm }\end{array}$
\end{tabular}




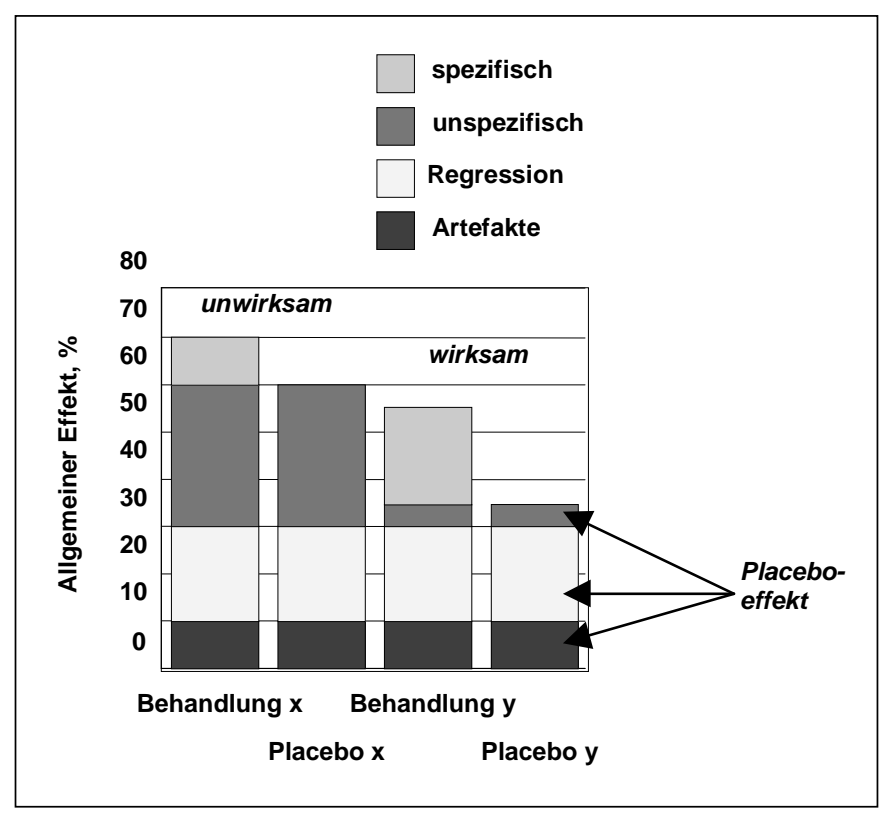

Abb. 1. Gedankenexperiment zum Wirksamkeitsparadox - Erläuterung siehe Text.

Effekte wirklich therapeutisch wertvoll sind. Das Gedankenexperiment macht die Konsequenz dieser Annahmen deutlich. Beide Voraussetzungen sind möglicherweise fatal und ziemlich sicher falsch. Die unspezifischen oder Placeboeffekte sollen bei der klassisch pharmakologischen Forschung natürlicherweise minimiert werden, weil man den Unterschied zwischen Verum und Placebo möglichst gross machen will, um den Wert des spezifischen Pharmakons zu belegen. Gerade diese unspezifischen Effekte sind aber möglicherweise die hauptsächlichen Träger des Therapieeffekts in der Praxis und sollten eigentlich in jeder effektiven Behandlung maximiert werden. Vor allem sind sie vielleicht je nach Therapieform unterschiedlich gross. Es gibt Daten, die zeigen, dass Placeboeffekte oder genauer, die Therapieeffekte in Placebogruppen, eben nicht gleicht gross sind [1], dass sie mit der Grösse der Verumeffekte variieren und relativ wenig mit dem Krankheitsbild [2-4], und vor allem, dass sie mit länger dauernden Studien eher zunehmen [4]. Und es gibt gute Gründe zu vermuten, dass Placeboeffekte oder unspezifische Therapieeffekte maximiert werden, wenn die Erwartungen der Patienten hoch sind, wenn Weltbild und Erklärungsmythos des Arztes mit denen des Patienten gut übereinstimmen [5]. Genau das ist aber bei komplementärmedizinischen Massnahmen in hohem Masse gegeben. Meistens wählen sich Patienten in komplementärmedizinischer Behandlung diese nach reiflicher Überlegung, nach fehlgeschlagenen konventionellen Behandlungsversuchen und oft nach langer Suche aus. Sie investieren also gehörig, nicht nur finanziell, sondern auch psychologisch und ideell. Von ihrem Selbstverständnis her sind komplementärmedizinische Behandlungsweisen meistens regulativ, d.h. sie versuchen, Selbstregulationsmechanismen wieder in Gang zu bringen. Die
Interventionen, die sie hierfür verwenden, sind von ihrer Therapietheorie her meist spezifisch. Aber wer sagt denn, dass die Effekte ebenso spezifisch sind? Möglicherweise sind komplementärmedizinische Massnahmen gute Wege, um auf unspezifischem Wege die Selbstheilungskräfte zu stimulieren. Möglicherweise tun sie das sehr effizient. Aber möglicherweise tun sie das auch vor allem vermittels eines grossen unspezifischen Therapieanteils. Immer vorausgesetzt, dies ist so, würde die Situation in Abbildung 1 die Problematik der komplementärmedizinischen Forschung gegenüber der konventionellen idealisiert wiedergeben. Wir haben guten Grund zu dieser Vermutung. Anekdotische Erfahrung von Forschern bestätigt genau dieses Szenario immer wieder. Eine Illustration liefert uns die jüngst publizierte Studie von Abbot et al. [6] zu geistigem Heilen als Therapie für chronische Krankheit (siehe auch den Journal Club im vorangegangenen Heft [7]). In dieser Studie erhielten 30 Patienten geistige Heilung, 30 Patienten erhielten eine Scheinbehandlung: Schauspieler beobachteten zunächst geistige Heiler bei ihrer Tätigkeit, um sie dann nachzuahmen und dabei von 1000 rückwärts zu zählen. Diese Art der Scheinbehandlung ist natürlich extrem glaubwürdig und maximiert damit selbstverständlich die unspezifischen Anteile der Behandlung. Es wundert einen denn auch nicht, dass diese Studie keine signifikanten Unterschiede zwischen echter und Scheinbehandlung zeigen konnte. Allerdings, und dies ist sehr interessant - Details hierzu im Kommentar des Journal Clubs [7] - waren die absoluten Effekte, die diese Behandlung erzeugte, also unspezifische plus spezifische Effekte, sehr gross. Leider haben die Autoren die Studie nicht von diesem Standpunkt aus diskutiert, sondern nur von jenem der spezifischen Unterschiede. Und von diesem gesehen sind die Unterschiede zwischen echter und Scheinbehandlung sehr gering.

Selbstverständlich ist es ein wichtiges Anliegen der Forschung herauszufinden, ob eine Behandlungsform insgesamt wirksam ist und ob sie eine Wirksamkeit besitzt, die über psychologische Komponenten hinausgeht. Aber es ist fatal, vor allem für Behandlungsformen wie denen der Komplementärmedizin, davon auszugehen, dass all die psychologischen und unspezifischen Effekte, die auch in Kontrollgruppen sehr deutlich sichtbar sind, unwichtig und in gewisser Weise Abfalleffekte sind. Umgekehrt: Diese Effekte sind die Basis, auf denen die spezifischen Effekte aufsitzen. Das Mittelalter verwendete eine wunderschöne Ikonographie für sein Verhältnis zur Tradition: Zwerge, die auf Schultern von Riesen sitzen [8]. In den Fenstern der Kathedrale von Chartres sind sie abgebildet. Johannes von Salisbury berichtet von seinem Lehrer Bernhard von Chartres, dass dieser zu sagen pflegte, wir, die Modernen, seien wie Zwerge, die auf Schultern von Riesen sitzen; deswegen sehen wir so weit. Dies ist ein gutes Bild für das Verhältnis von spezifischen zu unspezifischen Effekten: Die spezifischen Effekte sind wie Zwerge auf den Schultern von Riesen, deswegen reichen sie so weit. Manchmal kann es sinnvoll sein, nur die Grösse der Zwerge zu vermessen. Dies ist das, was placebokontrollierte Studien tun. Aber es ist reichlich kurz- 
sichtig zu meinen, man wisse dann etwas darüber, wie weit eine Therapieform reicht. Dazu muss man die Gesamtgrösse kennen: Zwerg plus Riese.

Was bedeutet dies, und was ist zu tun?

Dreierlei:

1. Es kann nicht angehen, dass man die spezifische Wirksamkeit zur alleinigen Richtschnur von Effektivität einer Therapieform macht.

2. Um die gesamte Effektivität zu kennen, benötigt man verschiedene Studienansätze, die sich komplementär ergänzen müssen: placebokontrollierte Studien, um die Grösse des spezifischen Effekts schätzen zu können; offene, randomisierte Vergleichsstudien, um die Wirksamkeit gegenüber Standardbehandlung schätzen zu können; vergleichende Studien an natürlichen Gruppen, um die Effekte in unkon- trollierten Praxis-Settings zu sehen; und schliesslich gross angelegte «Outcome»-Studien mit langen Beobachtungszeiten, um die Effekte in einer weitgestreuten Praxis zu dokumentieren. Erst alle Methoden zusammen ergeben das Mosaik der tatsächlichen Effekte einer Behandlungsmethode. 3. Es wird Zeit, dass wir uns um die Frage kümmern, was eigentlich die unspezifischen Therapieeffekte ausmacht. Möglicherweise sind gerade sie die gemeinsame Endstrecke sehr vieler therapeutischer Bemühungen. Möglicherweise sind sie auch die Brücke zu konventionellen Behandlungsweisen. Möglicherweise zeigt sich in ihnen, wie Selbstheilungsprozesse funktionieren. Dies müsste eigentlich nicht nur komplementärmedizinische, sondern alle Forscher interessieren.

H. Walach, Freiburg i. Br.

\section{Literatur}

1 Kleijnen J, de Craen AJM, Van Everdingen J, Krol L: Placebo effect in double-blind clinical trials: A review of interactions with medications. Lancet 1994:344: 1347-1349.

2 Kirsch I, Sapirstein G: Listening to Prozac but hearing placebo: A meta-analysis of antidepressant medications; in Kirsch I (ed): Expectancy, Experience, and Behavior. Washington, DC, American Psychological Association, 1999, pp 303-320.
3 Maidhof C, Dehm C, Walach H: Placebo response rates in clinical trials. A meta-analysis. Int $\mathbf{J}$ Psychol 2000;35:224(abstract).

4 Walach H, Maidhof C: Is the placebo effect dependent on time? in Kirsch I (ed): Expectancy, Experience, and Behavior. Washington, DC, American Psychological Association, 1999, pp 321-332.

5 Frank JD: Non-specific aspects of treatment: The view of a psychotherapist; in Shepherd M, Sartorius N (eds): Non-Specific Aspects of Treatment. Bern, Huber, 1989, 95-114.

6 Abbot NC, Harkness EF, Stevinson C, Marshall FP, Conn DA, Ernst E: Spiritual healing as a therapy for chronic pain: A randomized, clinical trial. Pain 2001;91: 79-89.

7 Journal Club. Forsch Komplementärmed Klas Naturheilkd 2001;8:170-180

8 Klibansky R: Standing on the shoulders of the giants. Isis 1936;26:147-149. 